Supporting Information 


\section{Design of Cobalt-Amine Complex as an Efficient Structure Directing Agent for One-Pot Synthesis of Co-SSZ-13 Zeolite}

Shichao Han, ${ }^{a}$ Xiaomin Tang, ${ }^{c}$ Ye Ma, ${ }^{a}$ Qinming Wu, ${ }^{*}, b$ Jiaqi Shi, ${ }^{a}$ Jian Li, ${ }^{a}$ Xiangju Meng, ${ }^{a}$ Anmin Zheng, ${ }^{c}$ and Feng-Shou Xiao ${ }^{*, b}$

${ }^{a}$ Key Lab of Applied Chemistry of Zhejiang Province, Department of Chemistry, Zhejiang University, Hangzhou, 310007, P. R. China.

${ }^{\mathrm{b}}$ College of Chemical and Biological Engineering, Zhejiang University, Hangzhou 310027, China.

${ }^{\mathrm{c}}$ State Key Laboratory of Magnetic Resonance and Atomic and Molecular Physics, National Center for Magnetic Resonance in Wuhan, Key Laboratory of Magnetic Resonance in Biological Systems, Wuhan Institute of Physics and Mathematics, Innovation Academy for Precision Measurement Science and Technology, Chinese Academy of Sciences, Wuhan, 430071, P. R. China.

KEYWORDS: designed synthesis, cobalt-amine complex, structure-directing agent, one-pot synthesis, $\mathrm{CH}_{4}-\mathrm{SCR}$ 


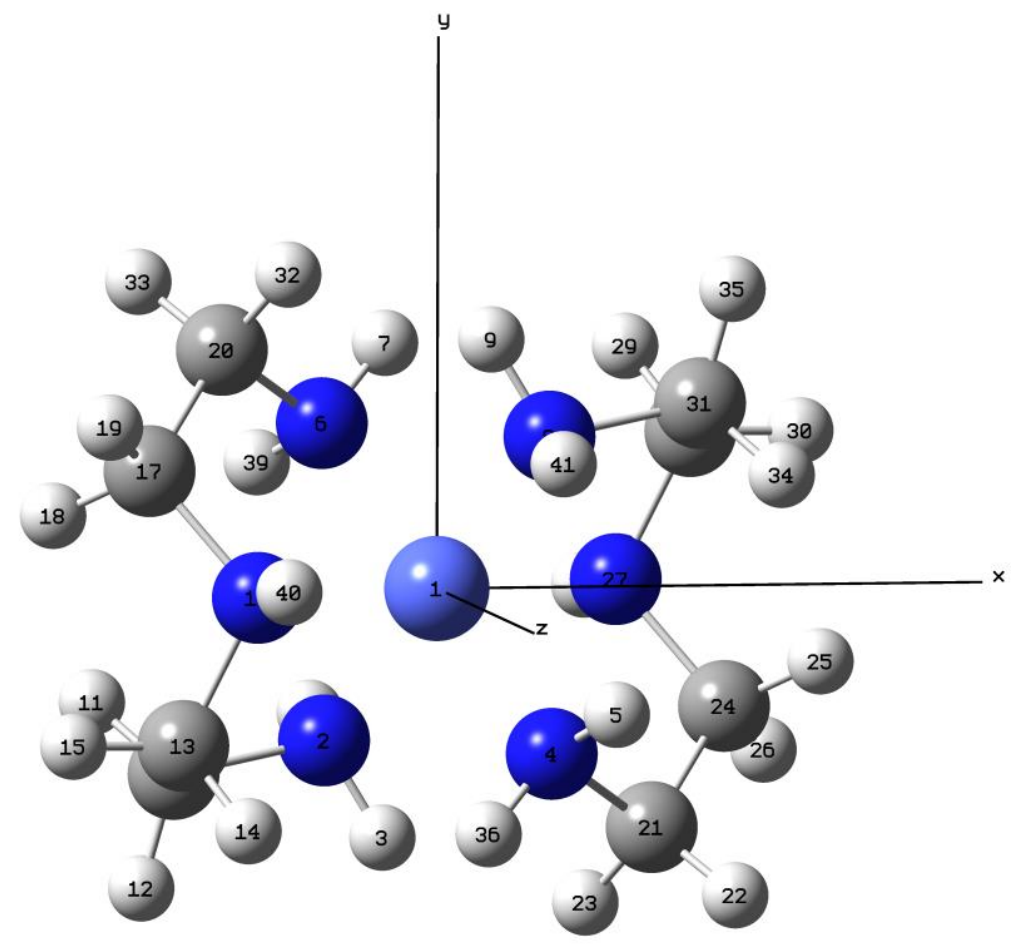

Figure S1. Optimized geometry of Co-DETA complex. 


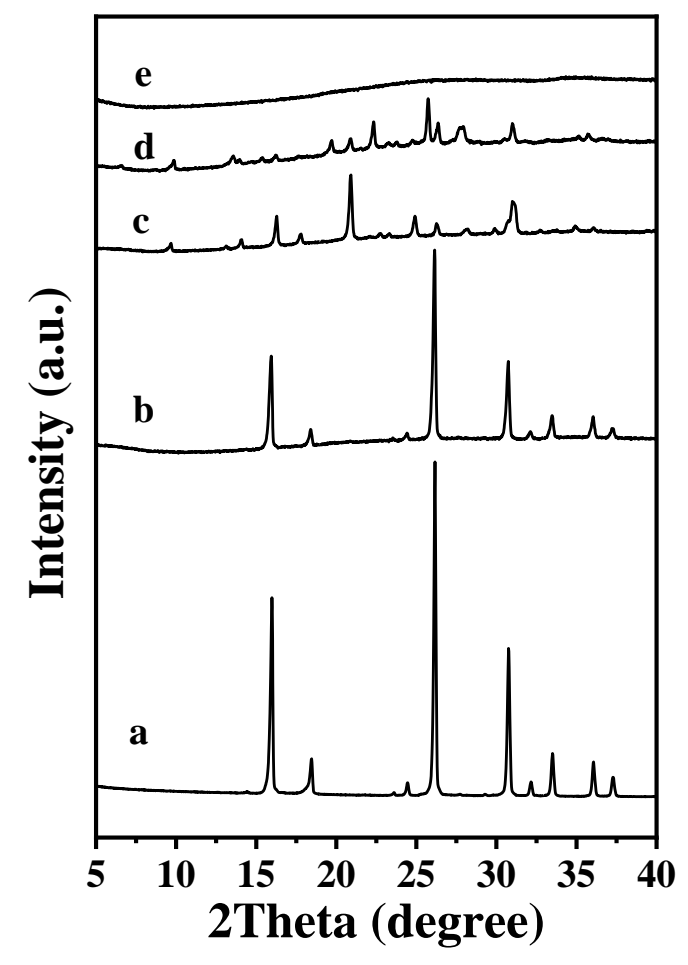

Figure S2. XRD patterns of the Co-SSZ-13 zeolite with $\mathrm{SDA} / \mathrm{SiO}_{2}$ ratios at (a) 0 , (b) 0.05 , (c) 0.12 , (d) 0.15 , and (e) 0.2 , respectively. 


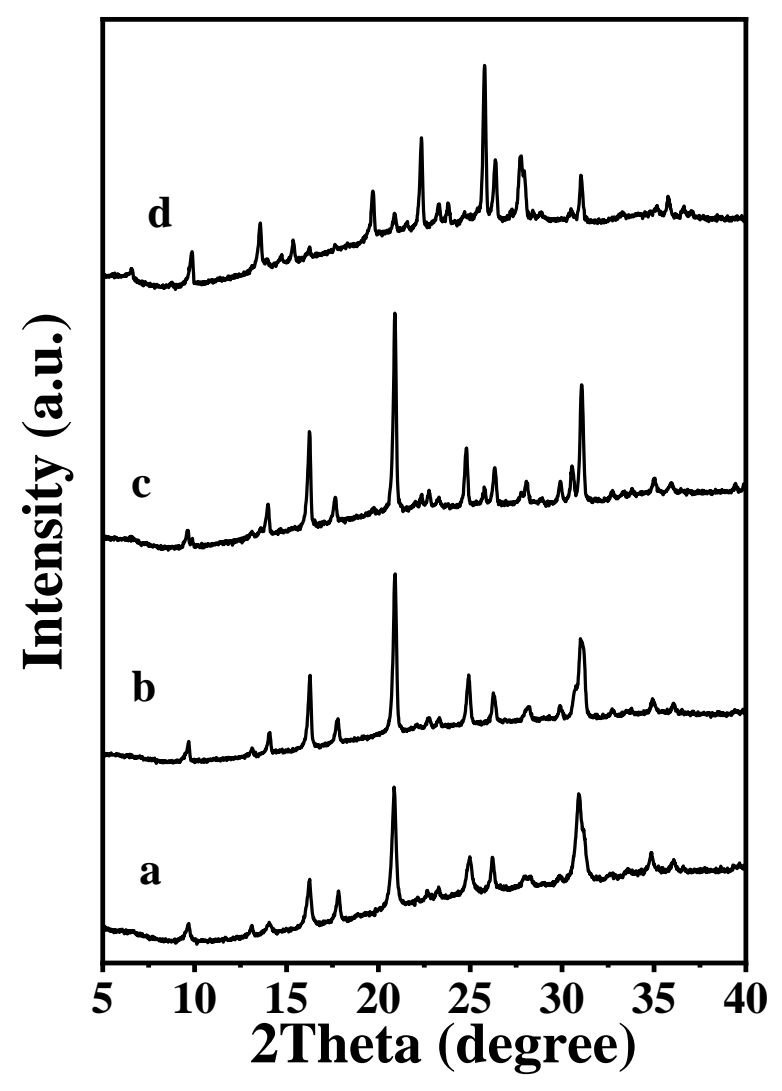

Figure S3. XRD patterns of the Co-SSZ-13 zeolite with $\mathrm{SiO}_{2} / \mathrm{Al}_{2} \mathrm{O}_{3}$ ratios of at (a) 20, (b) 24, (c) 30, and (d) 40, respectively. 


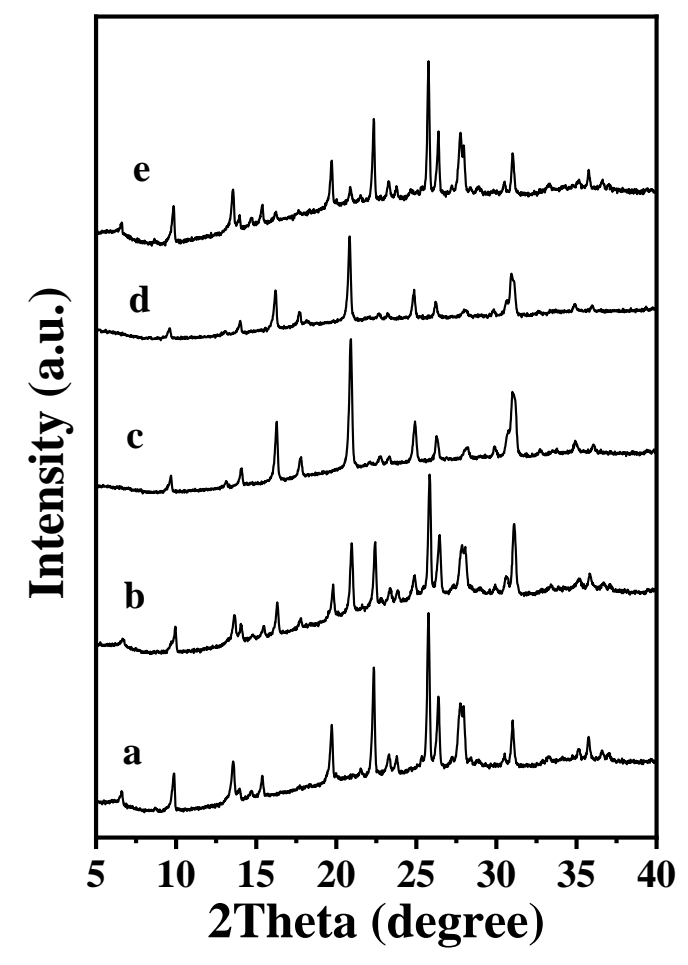

Figure S4. XRD patterns of the Co-SSZ-13 zeolite with $\mathrm{Na}_{2} \mathrm{O} / \mathrm{SiO}_{2}$ ratios at (a) 0.3, (b) 0.34 , (c) 0.37 , (d) 0.4 and (e) 0.45 , respectively. 


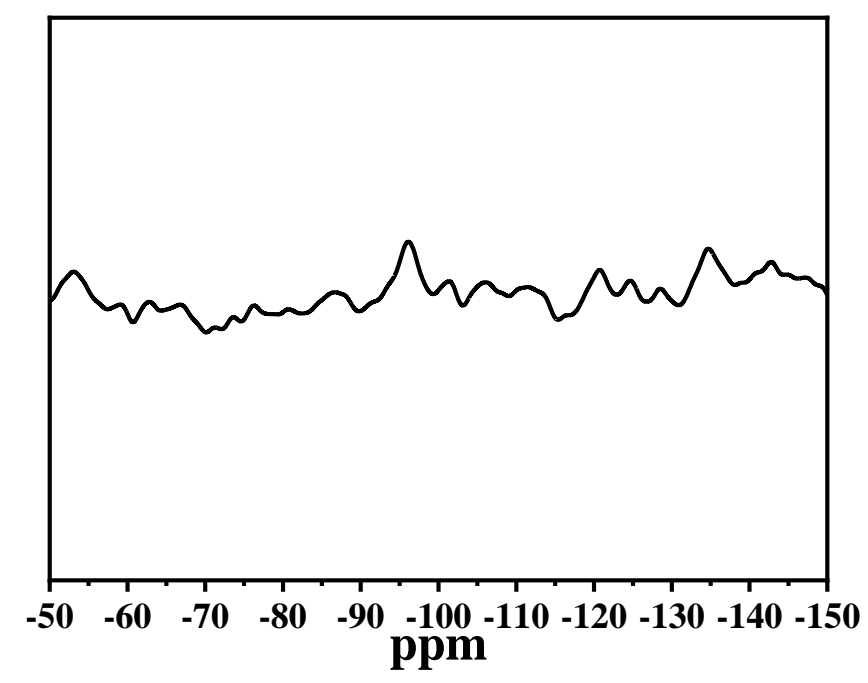

Figure S5. ${ }^{29}$ Si MAS NMR spectrum of the as-synthesized Co-SSZ-13 zeolite. 


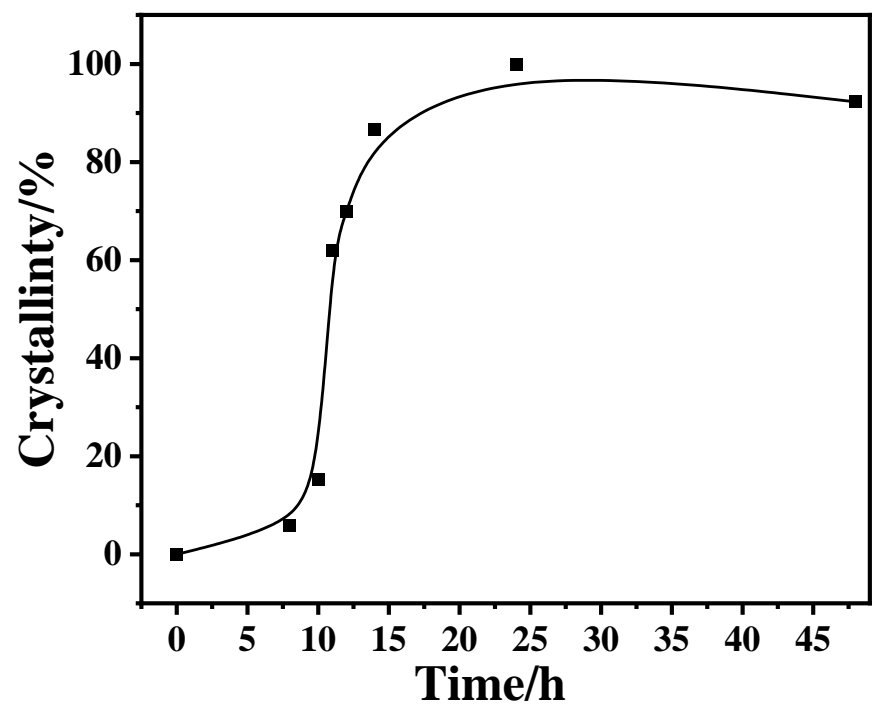

Figure S6. Dependence of the Co-SSZ-13 zeolite crystallinity on crystallization time. 

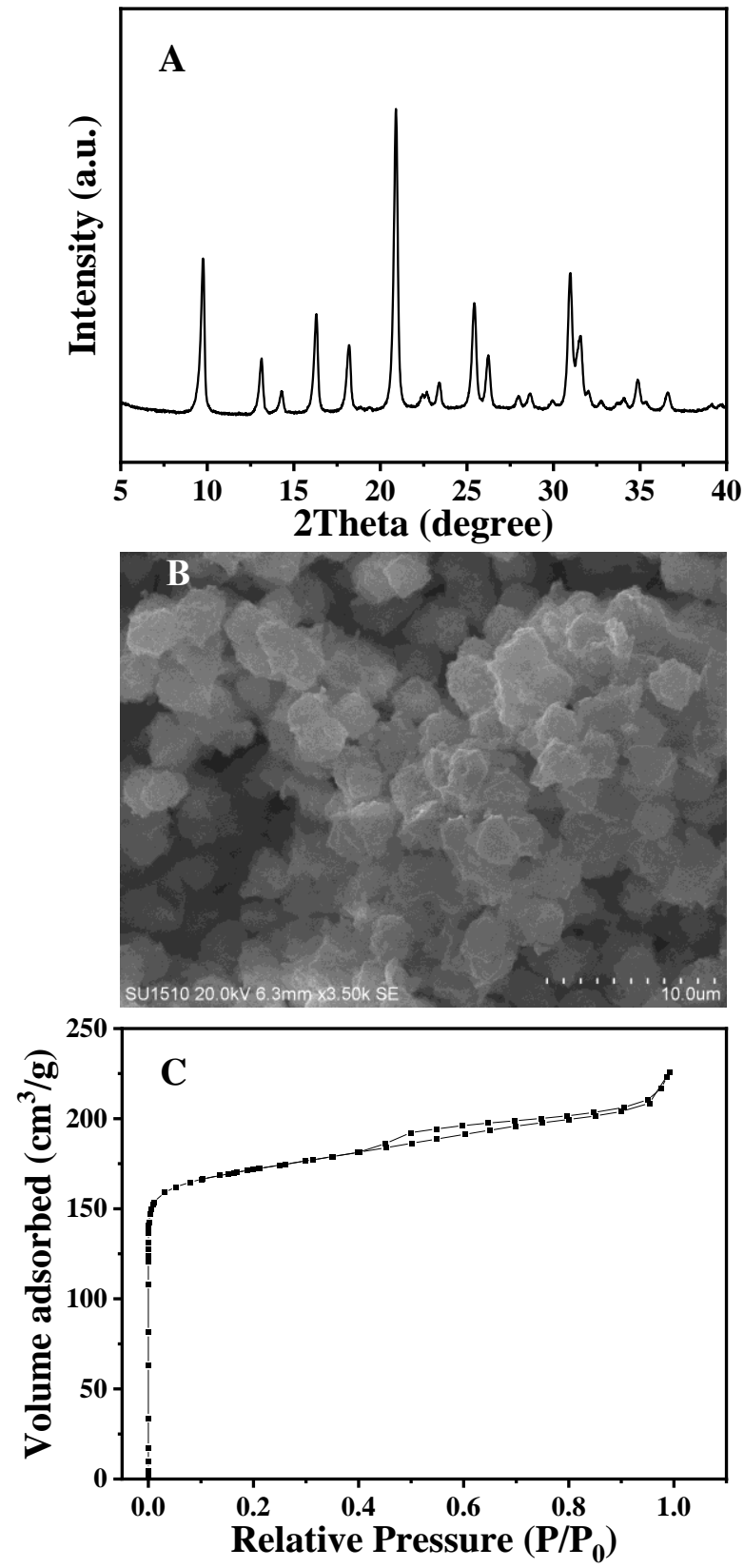

Figure S7. (A) XRD pattern, (B) SEM image, and (C) $\mathrm{N}_{2}$ sorption isotherm of the conCo-SSZ-13 zeolite.

Note: BET surface area and micropore volume of the con-Co-SSZ-13 zeolite are 542 $\mathrm{m}^{2} / \mathrm{g}$ and $0.29 \mathrm{~cm}^{3} / \mathrm{g}$, respectively. 
Table S1. ICP results of the as-synthesized and H-form Co-SSZ-13 zeolite.

\begin{tabular}{cccc}
\hline Sample & Si/Al & Co (\%) & Na (\%) \\
\hline as-synthesized & 4.69 & 11.36 & 2.79 \\
H-form & 5.12 & 4.38 & 0.011 \\
\hline
\end{tabular}


Table S2. Atom sites of optimized geometry of Co-DETA complex.

\begin{tabular}{|c|c|c|c|c|}
\hline Number & Atoms & $\mathbf{x}$ & $\mathbf{y}$ & $\mathbf{z}$ \\
\hline 1 & Co & -0.000022 & 0.000095 & -0.000065 \\
\hline 2 & $\mathrm{~N}$ & -0.826679 & -1.458055 & -1.128813 \\
\hline 3 & $\mathrm{H}$ & -0.310995 & -2.333812 & -1.012373 \\
\hline 4 & $\mathrm{~N}$ & 0.850094 & -1.41241 & 1.159111 \\
\hline 5 & $\mathrm{H}$ & 1.298033 & -0.996591 & 1.982465 \\
\hline 6 & $\mathrm{~N}$ & -0.850068 & 1.412705 & -1.159229 \\
\hline 7 & $\mathrm{H}$ & -0.201717 & 2.099518 & -1.558733 \\
\hline 8 & $\mathrm{~N}$ & 0.826564 & 1.458269 & 1.128643 \\
\hline 9 & $\mathrm{H}$ & 0.311578 & 2.334122 & 1.011089 \\
\hline 10 & $\mathrm{C}$ & -2.272898 & -1.713912 & -0.75075 \\
\hline 11 & $\mathrm{H}$ & -2.906754 & -1.11118 & -1.403919 \\
\hline 12 & $\mathrm{H}$ & -2.53441 & -2.760755 & -0.929199 \\
\hline 13 & $\mathrm{C}$ & -2.446869 & -1.338592 & 0.711815 \\
\hline 14 & $\mathrm{H}$ & -1.965886 & -2.061851 & 1.375661 \\
\hline 15 & $\mathrm{H}$ & -3.505044 & -1.309999 & 0.992561 \\
\hline 16 & $\mathrm{~N}$ & -1.804863 & 0.01178 & 0.922212 \\
\hline 17 & $\mathrm{C}$ & -2.71024 & 1.12828 & 0.414017 \\
\hline 18 & $\mathrm{H}$ & -3.479568 & 0.681121 & -0.217007 \\
\hline 19 & $\mathrm{H}$ & -3.224991 & 1.597001 & 1.256919 \\
\hline 20 & $\mathrm{C}$ & -1.908389 & 2.151825 & -0.377012 \\
\hline
\end{tabular}




\begin{tabular}{|c|c|c|c|c|}
\hline 21 & $\mathrm{C}$ & 1.908183 & -2.15179 & 0.376873 \\
\hline 22 & $\mathrm{H}$ & 2.554865 & -2.720852 & 1.052446 \\
\hline 23 & $\mathrm{H}$ & 1.418505 & -2.881463 & -0.270597 \\
\hline 24 & $\mathrm{C}$ & 2.709971 & -1.1286 & -0.414752 \\
\hline 25 & $\mathrm{H}$ & 3.480074 & -0.681831 & 0.215671 \\
\hline 26 & $\mathrm{H}$ & 3.223841 & -1.597615 & -1.25807 \\
\hline 27 & $\mathrm{~N}$ & 1.804866 & -0.011579 & -0.922324 \\
\hline 28 & $\mathrm{C}$ & 2.447282 & 1.338487 & -0.711118 \\
\hline 29 & $\mathrm{H}$ & 1.966537 & 2.062335 & -1.374569 \\
\hline 30 & $\mathrm{H}$ & 3.505463 & 1.309747 & -0.991753 \\
\hline 31 & $\mathrm{C}$ & 2.273164 & 1.713102 & 0.751621 \\
\hline 32 & $\mathrm{H}$ & -1.418921 & 2.881277 & 0.270893 \\
\hline 33 & $\mathrm{H}$ & -2.554921 & 2.721093 & -1.052555 \\
\hline 34 & $\mathrm{H}$ & 2.906345 & 1.109761 & 1.404771 \\
\hline 35 & $\mathrm{H}$ & 2.535264 & 2.759707 & 0.930634 \\
\hline 36 & $\mathrm{H}$ & 0.201756 & -2.099082 & 1.558908 \\
\hline 37 & $\mathrm{H}$ & 1.697441 & -0.141505 & -1.931935 \\
\hline 38 & $\mathrm{H}$ & -0.77767 & -1.290228 & -2.139749 \\
\hline 39 & $\mathrm{H}$ & -1.297768 & 0.997071 & -1.982756 \\
\hline 40 & $\mathrm{H}$ & -1.697487 & 0.142327 & 1.931765 \\
\hline 41 & $\mathrm{H}$ & 0.776386 & 1.291331 & 2.139824 \\
\hline
\end{tabular}


Table S3. Textural parameters of the as-synthesized and H-form Co-SSZ-13 zeolite.

\begin{tabular}{ccc}
\hline Sample & S BET $\left(\mathbf{m}^{\mathbf{2}} / \mathbf{g}\right)$ & VMicropore $_{\left(\mathbf{c m}^{3} / \mathbf{g}\right)}$ \\
\hline as-synthesized & 97 & 0.07 \\
H-form & 607 & 0.29 \\
\hline
\end{tabular}

